\title{
CERVICAL CANCER AND THE HUMAN IMMUNODEFICIENCY VIRUS: A REVIEW
}

Cynthia S Firnhaber, $M D, M S, D T M \& H$

Clinical HIV Research Unit, University of the Witwatersrand and Helen Joseph Hospital, Johannesburg

Pam Michelow, $M B$ BCh, $M S c$, MIAC

Cytology Unit, Department of Anatomical Pathology, National Health Laboratory Service and University of the Witwatersrand

Globally cervical cancer is one of the commonest cancers in women. It comprises approximately $12 \%$ of all cancers and is the commonest cancer in women in developing countries. The most recent compilation of global data indicates that an estimated 490000 new cases of cervical cancer occur annually worldwide and nearly $80 \%$ of these are in developing countries, where screening programmes are not well established and are poorly organised. Cervical cancer is the leading cause of cancer death among women. It is estimated that 270000 women die annually from cervical cancer, $85 \%$ of them in low-resource nations. ${ }^{1-3}$ In Africa, cervical cancer comprises $23.3 \%$ of all cancers in women. ${ }^{4}$ According to the South African National Cancer Registry (NCR) data, the lifetime risk for the development of cervical cancer in 1998 was 1 in 26 for South African women and 1 in 21 for black South African women. ${ }^{5}$ These are believed to be minimal rates, as the registry publishes only data collected from the pathology laboratories and is not a population-based cancer registry.

HIV/ AIDS poses a severe threat to global health. In addition, the HIV epidemic has hit hardest in regions of high prevalence of cervical dysplasia and cancer. The HIV epidemic in South Africa is one of the worst in the world. The prevalence of HIV among South African women attending antenatal clinics in 2006 was $29.1 \% .{ }^{6}$ With improved access to antiretroviral therapy, women are expected to live longer as the risk of death from opportunistic infections decreases. It is assumed that the incidence of cervical cancer and the prevalence of precursor lesions will increase, especially in countries that lack well-organised cervical screening. However, this remains to be seen.

\section{HUMAN PAPILLOMAVIRUS}

Cervical cancer and its precursor lesions are caused by infection with the human papillomavirus (HPV). The human papillomaviruses are part of the Papovaviridae family of viruses and consist of tightly coiled, circular, double-stranded DNA with about 8000 base pairs in their genome. The papillomavirus is an obligatory intranuclear virus that must infect mitotically active cells to institute infection. Within the cervix HPV most commonly infects the mitotically active transformation zone at the squamo-columnar junction, which explains in part why HPV is associated with both squamous and glandular neoplasia. Over 100 different HPV types have been identified, of which approximately 30 - 40 infect the anogenital tract. HPV viruses of the anogenital tract are divided into 'low-risk' and 'high-risk' types depending on their ability to produce neoplasia. ${ }^{7.8}$ There are a variety of opinions on how the human papillomavirus types should be classified. One commonly used classification (Munoz) published in 2003 divided them into 15 oncogenic (high-risk) types (HPV 16, 18, 31, 33, 35, $39,45,51,52,56,58,59,68,73,82)$ and 12 nononcogenic (low-risk) types (HPV 6, 11, 40, 42, 43, 44, $54,61,70$ and 72,81 and CP6108). ${ }^{9}$ The vast majority of HPV viral infections are cleared within 14 months in women with competent immune systems. However, through a complicated cascade of events some infections evade the immune system, becoming a persistent infection. Persistent HPV infection is the first step towards cervical dysplasia and cancer. The E6 and E7 proteins of oncogenic HPV types are known to facilitate the degradation of tumour suppression proteins. With the loss of suppression of these oncogenes, the cell cycle is disturbed. Factors that may increase the risk of persistent infection and cell dysregulation include immune suppression, cigarette smoke, multiple sexual partners, age of first intercourse, hormonal birth control and possibly other sexually transmitted infections such as Chlamydia., ${ }^{7.8}$

\section{RELATIONSHIP BETWEEN HIV AND HPV VIRUSES}

According to Denny et $a ._{.}{ }^{10}$ a strong relationship exists between two significant sexually transmitted viruses, HIV and HPV. Cervical cancer was made an AIDS-defining diagnosis by the Centers for Disease Control (CDC) in 1993. The natural history of cervical neoplasia is one of progression and regression, partially mediated by local cervical immunity. This process is not well understood. ${ }^{11}$ In HIV-positive women with cervical neoplasia, both pro-inflammatory and anti-inflammatory pathways in the cervical mucosa were found to be suppressed in comparison with the local mucosal en-

THE SOUTHERN AFRICAN JOURNAL OF HIV MEDICINE— JULY ZOOG 
vironment of HIV-negative women. ${ }^{12}$ HIV may increase the risk of HPV replication or transcription by a direct viral-viral interaction. ${ }^{13,14}$

\section{HPV IN HIV-SEROPOSITIVE WOMEN}

HPV oncogenic types 16 and 18 account for $90 \%$ of high-grade intraepithelial precursor lesions in HIV-negative women. ${ }^{15}$ However, data from a variety of studies in southern Africa show a diversity of HPV types. In Zambia Professor Parham in a 150-women study found that $98 \%$ of HIV-infected women harboured at least one type of HPV (85\% had a high-risk HPV type), with a median of four types per participant. HPV 52 was the most common. ${ }^{16}$ A study in South Africa of 148 HIV-infected women showed similar results, with 95\% of the women harbouring HPV, a median of three HPV types per participant, and 85\% of women having one or more oncogenic HPV types (HPV 16 accounted for 30\%, followed by 35 and 53). ${ }^{17}$ Data from other developing countries in South America and Asia also show a large diversity of oncogenic HPV types (including 16 and 18) in addition to other types, such as 33, 35, 52 and $81 .{ }^{18,19}$ Which oncogenic types are causing cervical cancer in HIV-seropositive women in Africa still needs to be determined. Recent results from a study in Kenya indicate that the prevalence of HPV 16 was similar in HIV-seropositive and HIV-seronegative women with invasive cervical cancer. $^{20}$

\section{CERVICAL LESIONS IN HIV-POSITIVE WOMEN}

Several studies have shown that invasive cervical cancer in HIV-positive women tends to present $10-15$ years earlier than in their HIV-negative counterparts. In addition, HIV-positive women with invasive cervical cancer have a much greater degree of immunosuppression than HIV-positive women without cervical cancer. Women with CD4 counts below 200 cells/ $\mu$ l are significantly more likely than HIV-negative women to have advanced-stage disease at presentation. ${ }^{21,22}$

HIV-positive women have higher rates of HPV and cervical abnormalities than HIV-negative women. A study by Denny et al. ${ }^{10}$ of 400 untreated HIV-infected women who underwent HPV DNA testing, cytology, colposcopy, histology and a CD4 count every 6 months for 36 months showed that $68 \%$ of women were positive for high-risk HPV DNA, 35\% had low-grade squamous intraepithelial lesions (LSIL) on Pap smear, and 13\% had high-grade squamous intraepithelial lesions (HSIL) on Pap smear.

Abnormal cytology and the presence of high-risk HPV DNA were strongly correlated with low CD4 counts and high viral loads. A study by Yamada and co-workers in Kenya ${ }^{23}$ demonstrated cervical HPV infection in $17 \%$ of
HIV-negative and 49\% of HIV-positive women. LSIL was found in 6.9\% HIV-negative and $21 \%$ of HIV-positive women, and HSIL in $0.6 \%$ of negative and $5.8 \%$ of positive women. HSIL was strongly associated with high-risk HPV types and low CD4 counts.

A study by Cardillo et al. ${ }^{24}$ showed HIV viral load to be significantly higher in women with cytological abnormalities. This study concluded that the degree of immunosuppression may contribute to the development of a squamous intraepithelial lesion, but once the lesion is established, disease progression may not be affected by CD4 counts. A Finnish study ${ }^{25}$ of 153 HIV-positive women followed up for a mean of 5.6 years showed $33 \%$ to have cervical neoplasia. The risk of cervical neoplasia was not associated with decreased CD4 counts, duration of HIV infection or use of antiretrovirals. ${ }^{25}$

The vast majority of LSIL regress spontaneously in immunocompetent women. HIV-positive women with LSIL have a lower rate of regression and more tendency to progression. In a study by Massad et al. ${ }^{26}$ progression of cervical dysplasia was observed in 14\% of HIV-positive women compared with 7\% of HIV-negative women. Regression to normal was noted in $43 \%$ of HIVpositive women and 66\% of HIV-negative women. In a study by Ahdieh et al., ${ }^{27}$ cervical dysplasia regressed in only $45 \%$ of HIV-positive women after a median of 2.7 years, which was significantly lower than the regression rate in HIV-negative women.

In summary, HIV-positive women tend to have a higher prevalence of HPV, more HPV types with multiple oncogenic-type infection, more HPV persistence and a higher prevalence of cytological abnormalities than their HIV-negative counterparts, and tend to present at an earlier age with cervical cancer.

\section{PREVENTION OF CERVICAL CANCER}

Prevention of cervical cancer can be primary or secondary. Primary prevention modalities include adopting safe sex practices and HPV vaccination. Secondary preventive (screening) techniques include cytology, visual inspection of the cervix and HPV testing.

\section{PRIMARY PREVENTION}

Sexual abstinence is the only way to completely prevent transmission of HPV. Transmission of HPV appears to be fairly easy. Correlated HPV types have been found on the external genitalia and cervix of women without any history of sexual intercourse and in reportedly monogamous couples. ${ }^{28-30}$ The rate of HPV transmission may be lower in circumcised males. ${ }^{31}$ The efficacy of condoms in preventing transmission of HPV is uncertain. One small study ${ }^{32}$ showed a decrease in the rate of genital HPV infection from 89.3/100 patient-years to $37.8 / 100$ patient-years in women reporting $100 \%$ 
condom use. Consistent use of condoms may increase HPV clearance and increase the rate of regression in HIV-negative women. ${ }^{32}$ The impact of condom use on HPV transmission in HIV-positive women is unknown.

Two vaccines that protect against certain types of HPV have recently become available. Cervarix (GlaxoSmithKline) produces antibodies to HPV types 16 and 18 while Gardasil (Merck) produces antibodies to HPV types 16, 18,6 and 11 (the latter two cause genital warts). ${ }^{33,34}$ Overseas trials have suggested that these two vaccines reduce the incidence of cervical cancer significantly, as it has been estimated that approximately $70 \%$ of cervical cancer is caused by HPV types 16 and 18 . However, cervical screening will still be necessary as $30 \%$ of cervical cancers are caused by HPV types other than 16 and 18 . Also, it will be required for women who have not received the vaccine.

The prevalence of HPV types in southern Africa as discussed above may differ from those overseas, so data from HPV vaccination studies elsewhere may not be as helpful in southern Africa. The safety and efficacy of the preventive HPV vaccines in HIV-positive women also remains to be determined, as it is not known whether a sufficient antibody response to the vaccine will be obtained. Ideally girls should be vaccinated before risk of exposure to either HIV or HPV. In addition, HIV-positive women have a higher rate of infection with multiple HPV types, which may impact negatively on the efficacy of current vaccines against HPV types 16 and 18. Another type of HPV vaccine that is still in the early phases of testing is the therapeutic vaccine, to be given to women already infected with HPV to block the E6 and E7 oncogenic proteins and thus prevent progression. ${ }^{35}$

\section{SCREENING FOR CERVICAL CANCER}

Cervical screening programmes are traditionally based on cervical smears. In countries with well-organised cytologically based cervical screening programmes (such as Mexico, Columbia and many developed nations), the reduction in the incidence of and mortality from cervical cancer has been dramatic. ${ }^{36,37}$ A cervical smear-based screening programme is a three-step (visits) approach, viz. women have a smear taken at a clinic, women with abnormal smears then have to be referred to a colposcopy centre for colposcopic biopsy to confirm the cervical results, and then, once confirmed, women have to be referred for treatment of the lesion.

Cytology is associated with a significant false-negative rate. The estimated sensitivity is only $50-60 \%$ for detection of cervical intra-epithelial neoplasia HSIL or greater in routine screening settings. The range of sensitivities and specificities of conventional cytology for the detection of HSIL in screening studies are 40 - 86\% and 88 - 99\%, respectively. ${ }^{38-41}$ Despite these limitations, cervical cytology remains the only proven method for reducing the incidence and mortality of cervical cancer in large-scale population screening.

South Africa's cervical screening programme includes three smears in a lifetime at 10-year intervals starting at age 30. If this could be undertaken effectively, it is estimated that $66 \%$ of invasive cervical cancer would be eliminated. ${ }^{42}$ Although South Africa's screening programme was launched in 2000, it has yet to be implemented on any large scale. A cytologically based cervical screening programme requires a reasonably well-functioning health care system. Unless good follow-up and referral mechanisms are in place, women may be lost to follow-up at any one of these steps. Accessibility of colposcopy and treatment of cervical dysplasia is currently very limited in the South African public sector.

Visual inspection of the cervix with acetic acid (VIA) has been investigated as an alternative to cytology in low-resource nations. VIA is a simple procedure involving swabbing the cervix with 5\% acetic acid and after a few minutes observing any change of colour of a normal pink cervix to white. The white areas may represent cervical dysplasia. The normal columnar epithelium is dark pink to red. If in the area of the mitotically active transition zone, the white areas may be indicative of cervical dysplasia and can be treated with cryotherapy at the same visit. ${ }^{43}$ The convenience and relative simplicity of VIA makes it preferable to the Pap smear in many resource-limited settings, because it does not require the client to return to the clinic for her results.

Assessment of VIA accuracy in large cross-sectional, randomised controlled trials in developing countries in HIV-negative women indicates that its sensitivity in detecting high-grade precancerous lesions ranges from $66 \%$ to $96 \%$ (median 84\%), its specificity from 64\% to $98 \%$ (median $82 \%$ ), its positive predictive value from $10 \%$ to $20 \%$ and its negative predictive value from $92 \%$ to $97 \% .{ }^{44,45}$ The major strengths of VIA include its simplicity: there is no need to prepare the patient; it can be taught to nurses, nurse-midwives and other health workers in a short space of time; it costs less than other approaches in routine use; there is real-time availability of results (results are available immediately, eliminating the need for multiple visits in most cases, and reducing loss to follow-up); there is potential for immediate linkage with investigation/treatment; and no specimen transport, expensive laboratory equipment or highly trained personnel are needed.

The major limitations of VIA include low specificity (generally less than 85\%) - a considerable number of women who test positive do not have disease, resulting in excessive diagnosis and treatment, and unnecessary anxiety; 
lack of standardised methods of quality control (there is no permanent record of the test that can be reviewed later); training and competency of screeners are difficult to evaluate; its ability to detect endocervical lesions is limited as a result of difficulties in sampling and visualising the endocervical canal, as well as less experience among readers in recognising glandular cell lesions; and tests to follow up women who have been treated are lacking. Finally, the efficacy and cost-effectiveness of VIA-based population screening programmes in reducing the incidence of, and mortality from, cervical cancer are not known and remain to be established, as do the long-term complications of over-treatment. ${ }^{43-45}$ VIA has mostly been evaluated as an once-in-a-lifetime screening test, and its performance in periodic screening has not been assessed. Validation in HIV-positive women is required.

The vast majority of HPV infections will clear spontaneously, especially in women below the age of 30 years, so HPV DNA testing has good negative predictive value but less good positive predictive value. Other disadvantages of HPV DNA testing are the cost, dependence on reagents currently produced by very few commercial manufacturers, and low specificity in younger women. HPV testing usually requires sophisticated laboratories with highly trained personnel, often not available in low-resource nations. In addition, samples for HPV tests need to be transported to the laboratory and patients need to return to the clinic for results, so there is potential loss to follow-up. ${ }^{46-48} \mathrm{~A}$ rapid HPV test for use in low-resource countries, the HPV Digene Fast Test, has recently been developed. It obviates the need for sophisticated laboratories, and one study has shown good results. ${ }^{49}$ Further investigations in this regard are required, especially in HIV-seropositive women.

Various combinations of cytology, VIA and HPV testing have been described, but the best method of screening in both HIV-positive and HIV-negative women has yet to be determined. ${ }^{50,51}$

\section{TREATMENT OF CERVICAL DYSPLASIA}

If colposcopy has confirmed the Pap smear cytology result of a high-grade lesion with histological results of CIN 2 or CIN 3, treatment of the lesion is required. Treatment can be done via a variety of methods. A very efficient outpatient procedure requiring only local anaesthesia is called either LEETZ (loop electrosurgical excision of the transition zone) or LEEP (loop electrosurgical excision procedure) biopsy. The procedure uses a thin electrified wire to remove the lesion up to $7 \mathrm{~mm}$ in depth from the transitional zone. LEEP or LEETZ is a very effective procedure in non-HIV-seropositive women, with a success rate of $80-85 \% .{ }^{44,52}$ However, a study in Soweto indicated that in HIV-seropositive women in South Africa the failure rate may be as high as $50 \%$ (defined as incomplete margins on pathological specimens). ${ }^{53}$ Close follow-up is required in HIV seropositive women and additional procedures may be needed.

More definitive treatment includes the cone biopsy. This is an effective mode of treatment, but requires referral to a centre with a skilled gynaecologist, inpatient admission, and operating room availability. ${ }^{52}$

Cryotherapy can be done after the VIA procedure, during the same visit as discussed above, eliminating the anxiety and loss to follow-up that lengthy delays in follow-up visits cause and reducing the inconvenience of work, child-care and transportation issues. The procedure needs a consistent supply of nitrous oxide with a cryotherapy gun and probe. Freezing the lesion requires application of the gas for about 2 minutes and the patient should come for follow-up visits at 6 weeks, 6 months and 12 months (Gosbeck Parham, personal communication). Recurrent lesions found after VIA are referred for LEEP. Cryotherapy means that there are no pathology results, so it is not known what lesion was treated. Large lesions and lesions spreading into the cervical canal cannot be treated with cryotherapy and need to be referred for LEEP. ${ }^{43}$

Hysterectomy is reserved for refractory cases of carcinoma in situ that cannot be treated appropriately with the above methods.

\section{OTHER TREATMENT MODALITIES}

Micronutrients, vitamins (vitamins $A, E$ and $C$ ) and antiretroviral agents (gancyclovir and cidofovir) have not shown any positive effect in the treatment of cervical dysplasia and should not be recommended. ${ }^{52}$ As HPV infection is an immunomodulated disease, there is some hope that HIV antiretroviral therapy (ART) would improve regression rates and prevent the progression of cervical dysplasia as ART has improved the outcomes of patients suffering from Kaposi's sarcoma. ${ }^{54}$ However, findings of reversal of cervical dysplasia in HIV-positive women receiving ART have been quite controversial, with disparate results. ${ }^{55,56}$ Reports indicate that antiretroviral drugs may improve outcomes in cervical dysplasia in HIV-infected women. However, other cohort studies show that ART has no effect on cervical dysplasia. ${ }^{57}$ As HPV is already integrated into the host genome and oncogenic gene changes are already occurring, ART given after the lesions have been developed may be too late to change the course of the HPV disease progression. Larger prospective cohort studies are needed to assess the effectiveness of ART in preventing cervical cancer in women with HPV-HIV coinfection.

With the effective ART roll-out in South Africa many women will survive opportunistic diseases, and cervical cancer screening needs to be on the agenda of HIV/ART 
clinics. With the overwhelmed public sector obstetrics and gynaecology services in South Africa, limited accessibility, long waiting periods between Pap smears, colposcopy and LEEP, disjointed care and loss to follow-up between departments, cervical cancer screening and treatment need to be available and offered in HIV clinics as an integrated service.

\section{REFERENCES}

1. Ferlay J, Bray F, Pisani P, Parkin DM. International Agency for Research on Cancer (IARC). GLOBOCAN 2002: Cancer Incidence, Mortality and Prevalence Worldwide. Lyon, France: IARCPress; 2004; Cancer Base No. 5, version 2.0.

2. Parkin D, Whelan S, Ferlay J, et al., eds. Cancer Incidence in Five Continents. Vol. VIII. Lyon: IARC Press, 2002 ( IARC Scientific Publications No. 155).

3. Steward BW, Kleihues P. World Cancer Report. Lyon: IARC Press, 2003.

4. Parkin D, Sitas F, Chirenje M, Stein L, Abratt $R$, Wabinga H. Cancer in indigenous Africans - burden, distribution and trends. Lancet Oncol 2008; 9: 683-692.

5. Mqogi $N$, Kellet $P$, Sitas $F$, Jula $M$. Incidence of histologically diagnosed cancer in South Africa, 1998-1999. National Cancer Registry Report. Johannesburg: National Health Laboratory Service, 2004: 116.

6. Summary of Biennial Report on the State of the South African HIV/AIDS Epidemic. South African Department of Health Study, 2006. www.doh.gov.za/docs/reports (accessed 28 December 2008).

7. Shew $M$, Fortenberry $D$, Wanzhu $T$, et al. Association of condom use, sexual behaviours, and sexually transmitted infections with the duration of genital human papillomavirus infection among adolescent women. Arch Pediatr Adolesc Med 2006; 160: 151-156.

8. IAC Task Force. Human papillomavirus. Acta Cytol 1998; 42: 50-58.

9. Muñoz N, Bosch FX, de Sanjosé $S$, et al. Epidemiologic classification of human papillomavirus types associated with cervical cancer. NEng/ J Med 2003; 348: 518527.

10. Denny $L$, Boa R, Williamson $A$, et al. Human papillomavirus infection and cervical disease in human immunodeficiency virus-1 infected women. Obstet Gynecol 2008; 111: 1380-1387.

11. Stanley M. Immune responses to human papillomavirus. Vaccine 2006; 24: suppl 1, S16-S22.

12. Kobayashi $A$, Greenblatt $R$, Anastos $K$, et al. Functional attributes of mucosal immunity in cervical neoplasia and effects of HIV infection. Cancer Res 2004; 64: 6766-6774.

13. Dolei $A$, Curreli $S$, Marongui $P$, et al. Human immunodeficiency virus infection in vitro activates naturally integrated human papillomavirus type 18 and induces synthesis of L1 capsid protein. J Gen Virol 1999; 80: 2937-2944.

14. Vernon S, Hart C, Reeves W, Icenogle J. The HIV-1 Tat protein enhances E2dependant human papillomavirus 16 transcription. Virus Res 1993; 27: 133-145.

15. Smith JS, Lindsay L, Hoots $B$, et al. Human papillomavirus type distribution in invasive cervical cancer and high grade cervical lesions: A meta-analysis update. Int J Cancer 2007; 121: 621-632.

16. Parham GP, Sahasrabuddhe $W$, Mwanahamuntu MH, Shepherd BE, Hicks ML, Stringer EM. Prevalence and predictors of squamous intraepithelial lesions of the cervix in HIV-infected women in Lusaka, Zambia. Gynecol Oncol 2006; 103(3): 10171022.

17. Firnhaber $C$, Zungu $K$, Williamson $A$, et al. Diverse and high prevalence of human papillomavirus associated with a significant high rate of cervical dysplasia in HIVinfected women in Johannesburg. Acta Cytol 2009; 53: 10-18.

18. Cerqueira DM, Moraes DD, Camara GN, et al. High HPV genetic diversity in women infected with HIV-1 in Brazil. Arch Virol 2007; 152(1): 75-83.

19. Bollen $\amalg$, Chuachoowong $R$, Kilmarx PH, et al. Human papillomavirus (HPV) detection among human immunodeficiency virus-infected pregnant Thai women: implications for future HPV immunization. Sex Transm Dis 2006; 33(4): 259-264.

20. De Vuyst $H$, Gichangi $P$, Estambale $B$, Njuguna $E$, Franceschi $S$, Temmerman M. Human papillomavirus types in women with invasive cervical carcinoma by HIV status in Kenya. Int J Cancer 2008; 122: 244-246.

21. Lomalisa $P$, Smith $T$, Guidozzi $F$. Human immunodeficiency virus infection and invasive cervical cancer in South Africa. Gynecol Oncol 2000; 77: 460-463.

22. Moodley M, Moodley J, Kleinschmidt I. Invasive cervical cancer and human immunodeficiency virus infection: a South African perspective. Int J Gynecol Cancer 2001; 11(3): 194-197.

23. Yamada $R$, Sasagawa $T$, Kirumbi L, Kingoro A, Karanja D, Kiptoo M. Human papillomavirus infection and cervival abnormalities in Nairobi, Kenya, an area with a high prevalence of human immunodeficiency virus infection. J Med Virol 2008; 80: 847-855.

24. Cardillo M, Hagan $R$, Abadi J, Abadi M. CD4 T-cell count, viral load and squamous intraepithelial lesions in women infected with the human immunodeficiency virus. Cancer (Cancer Cytopathol) 2001; 93: 111-114.

25. Lehtovirta $\mathrm{P}$, Paavonen J, Heikinheimo 0. Risk factors, diagnosis and prognosis of cervical intraepithelial neoplasia among HIV-infected women. Int J STD AIDS 2008; 19: 37-41.

26. Massad $S$, Ahdieh L, Benning L, et al. Evolution of cervical abnormalities among women with HIV-1: evidence from surveillance cytology in the WH study. J Infect Dis 2001; 27: 432-442.

27. Ahdieh $L$, Li R, Levine $A$, Massad $L$, et al. Highly active antiretroviral therapy and cervical squamous intraepithelial lesions in human immunodeficiency virus-positive women. J Nat/ Cancer Inst 2004; 96: 1070-1076.
28. Winer $R$, Lee $S$, Hughes J, et al. Genital human papillomavirus infection: incidence and risk factors in a cohort of female university students. Am J Epidemiol 2003; 157: 218-226.

29. Moscicki A. Impact of HPV infection in adolescent populations. J Adolesc Health 2005; 37 : 6 suppl, S3-S9.

30. Castellsague $X$, Bosch $F_{1}$ Munoz $N$, et al. Male circumcision, penile human papillomavirus infection and cervical cancer in female partners. N Engl J Med 2002. 346: 1105-1112

31. Winer $R$, Hughes J, Feng 0 , et al. Condom use and the risk of genital human papillomavirus infection in young women. N Engl J Med 2006; 354: 2642-2654.

32. Holmes $K$, Levine $R$, Weaver $M$. Effectiveness of condoms in preventing sexually transmitted infections. Bull World Health Organ 2004; 82: 454-461.

33. Harper D, Franco EL, Wheeler $C$, et al. Efficacy of a bivalent L1 virus-like particle vaccine in prevention of infection with human papillomavirus types 16 and 18 in young women: a randomised controlled trial. Lancet 2004; 364: 1757-1765.

34. Villa LL, Costa RL, Petta CA, et al. Prophylactic quadrivalent human papillomavirus (types 6, 11, 16, and 18) L1 virus-like particle vaccine in young women: a randomised double-blind placebo-controlled multicentre phase II efficacy trial. Lancet Oncol 2005; 6(5): 271-278.

35. De Jong A, O'Neil T, Khan A, et al. Enhancement of human papillomavirus (HPV) type 16 E6 and E7 specific T cell immunity in healthy volunteers through vaccination with TA-CIN, an HPV 16 L2E7E6 fusion protein vaccine. Vaccine 2002; 20: 34563464

36. Aristizabal N, Cuello C, Correa P. The impact of vaginal cytology on cervical cancer risks in Cali, Columbia. Int J Cancer 1984; 34: 5-9.

37. Lazcano-ponce E, Palacio-Meijia L, Allen-Leigh B, et al. Decreasing cervical cancer mortality in Mexico: effect of Papanicoloau coverage, birth rate and the importance of diagnostic validity of cytology. Cancer Epidemiol Biomarkers Prev 2008; 17: 28082817.

38. Nanda K, McCrory DC, Myers ER, et al. Accuracy of the Papanicolaou test in screening for and follow-up of cervical cytological abnormalities: A systematic review. Ann Intern Med 2000; 132: 819.

39. Bastian L, Datta S, Hasselbald V, Hickey J, Myers E, Nanda K. Evaluation of Cervical Cytology, 5. Rockville, MD: Agency for Health Care Policy and Research (AHCPR), 1999. Evidence report/technology assessment prepared by Duke University under contract 290-97-0014. AHCPR puplication 99-E010. hstat nlm nih gov/hq/Hquest/ $\mathrm{db} /$ local epc er cyt/screen/DocTitle/s48139 (accessed 25 February 2003).

40. Fahey MT, Irwig L, Macaskill P. Meta-analyses of Pap test accuracy. Am J Epidemiol 1995; 141: 680-689.

41. Mannino JR. Natural history of false-negative Papanicolaou smears: A prospective study using screening colposcopy in addition to cytology. J Am Osteopath Assoc 1998; 98: 546.

42. Sankaranarayanan $R$, Budukh $A$, Rajkumar R. Effective screening programmes for cervical cancer in low and middle income developing countries. Bull World Health Organ 2001; 79: 954-962.

43. Carr K, Sellors J. Cervical cancer screening in low resource settings: using visual inspection with acetic acid. J Midwifery Womens Health 2004; 49(4): 329-337.

44. Sankaranarayanan $R$, Gaffikin L, Jacob M, Sellors J, Robles S. A critical assessment of screening methods for cervical neoplasia. Int J Gynecol Obstet 2005: 89: 504-512.

45. Miller $B A$, Nazeer $S$, Fonn $S$, et al. Report on consensus conference on cervical cancer screening and management. Int $J$ Cancer 2000; 86: 440-447.

46. Wright TC, Schiffman M, Solomon D, et al. Interim guidance for the use of human papilloma virus DNA testing as an adjunct to cervical cytology for screening. Obstet Gynecol 2004; 103: 304-309.

47. Howard M, Sellors J, Kaczorowski J. Optimizing the hybrid capture II human papillomavirus test to detect cervical intraepithelial neoplasia. Obstet Gynecol 2002 100: 980-982.

48. Sellors J. HPV in screening and triage: towards an affordable test. HPV Today 2005 8: 4-5.

49. Qiao $Y$, Sellors J, Eder $P$, et al. A new HPV-DNA test for cervical cancer screening in developing regions: a cross-sectional study of clinical accuracy in China. Lancet Oncol (in press).

50. Goldie S, Gaffikin L, Goldhaber-Fiebert J, et al. Cost-effectiveness of cervical cancer screening in five developing countries. N Engl J Med 2005; 353: 2158-2168.

51. Mandelblatt J, Lawrence W, Gaffikin L, et al. Costs and benefits of different strategies to screen for cervical cancer in less-developed countries. J Natl Cancer Inst 2002; 94: 1469-1483.

52. Cox MD. Management of precursor lesions of cervical carcinoma: history, host defense, and a survey of modalities. Obstet Gynecol Clin North Am 2002; 29: 751 758.

53. Adam $Y$, van Gelderen $C_{1}$ de Bruyn $G$, et al. Predictors of persistent cytologic abnormalities after treatment of cervical intraepithelial neoplasia in Soweto, South Africa: A cohort study in a HIV high prevalence population. BMC Cancer 2008; 8 : 211.

54. Nasti G, Marellotta F, Berretta $M$, et al. Impact of highly antiretroviral therapy on the presenting features and outcome of patients with acquired immunodeficiency syndrome-related Kaposi sarcoma. Cancer 2003; 98(11): 2440-2446.

55. Heard I, Tassie JM, Kazatchikine $M$, et al. Highly active antiretroviral therapy enhances regression of cervical intraepithelial neoplasia in HIV-seropositive women. AIDS 2002: 16: 1799-1802.

56. Minkoff $H$, Ahdieh $L$, Massad LS, et al. The effect of highly active antiretroviral therapy on cervical cytologic changes associated with oncogenic HPV among HIVinfected women. AIDS 2001; 15: 2157-2164.

57. Lillo $F_{1}$ Ferrari $D_{1}$ Veglia $F_{1}$ et al. Human papillomavirus infection and associated cervical disease in human immunodeficiency virus-infected women: effect of highly active antiretroviral therapy. J Infect Dis 2001; 184: 547-551. 